\title{
Identification of Very Red Counterparts of SiO Maser and OH/IR Objects in the GLIMPSE Survey
}

\author{
Shuji Deguchi \\ Nobeyama Radio Observatory, National Astronomical Observatory \\ Minamimaki, Minamisaku, Nagano 384-1305, Japan \\ Jun-ichi NAKASHIMA \\ Academia Sinica, Institute of Astronomy and Astrophysics, PO Box 23-141, Taipei, 106, Taiwan \\ Sun KwoK \\ Department of Physics, University of Hong Kong, Hong Kong, China \\ and \\ Department of Physics 83 Astronomy, University of Calgary, Calgary, Alberta, Canada T2N 1N4 \\ Nicolas KONING \\ Department of Physics $\&$ Astronomy, University of Calgary, Calgary, Alberta, Canada T2N 1N4 \\ (ApJ part 1, August 10, 2007, v665 no.1 issue in press)
}

\begin{abstract}
Using the 3.6/4.5/5.8/8.0 $\mu \mathrm{m}$ images with $1.2^{\prime \prime}$ pixel resolution from the Spitzer/GLIMPSE survey, we investigated 23 masing and 18 very red objects that were not identified in the 2MASS survey. Counterparts for all selected objects were found in the GLIMPSE images. Color indices in these IR bands suggest the presence of a high-extinction layer of more than a few tenths of a solar mass in front of the central star. Furthermore, radio observations in the $\mathrm{SiO}$ and $\mathrm{H}_{2} \mathrm{O}$ maser lines found characteristic maser-line spectra of the embedded objects, e.g., the $\mathrm{SiO} J=1-0$ line intensity in the $v=2$ state stronger than that of the $v=1$ state, or very widespread $\mathrm{H}_{2} \mathrm{O}$ maser emission spectra. This indicates that these objects are actually enshrouded by very thick circumstellar matter, some of which cannot be ascribed to the AGB wind of the central star. Individually interesting objects are discussed, including two newly found water fountains and an $\mathrm{SiO}$ source with nebulosity.
\end{abstract}

Subject headings: infrared: stars — stars: AGB and post-AGB — stars: mass loss — radio lines: stars

\section{Introduction}

The discovery of stellar maser sources (Wilson \& Barre 1968) and their association with very red infrared objects has led to a new class of objects called $\mathrm{OH} / \mathrm{IR}$ stars. From the $\mathrm{OH}$ profiles, we came to the realization that the maser emissions are a manifestation of the mass loss process (Kwok 1976), and $\mathrm{OH} / \mathrm{IR}$ stars are likely to be stars in the asymptotic giant branch (AGB) phase of evolution (Habing 1996; Sevenster et al. 2002). The mass loss process also creates a thick circumstel- 
lar dust envelope which often obscures the central star. Although $\mathrm{OH} / \mathrm{IR}$ stars are often bright midinfrared objects (Jiménez-Esteban et al. 2006), many of them have no optical or near infrared counterparts. In the absence of a spectral classification based on photospheric spectroscopy, the nature of these stars is classified based on the circumstellar spectra in the mid-infrared (Kwok, Volk, \& Bidelman 1997).

It should be noted that the mid-infrared colors alone are not enough to determine the nature of maser sources. For example, the IRAS colors of mass-losing evolved stars overlap with colors of young stellar objects embedded in molecular clouds (van der Veen \& Habing 1988), and there is no well-defined boundaries in the colorcolor diagram between young and evolved stars (Weintraub 1990). The maser characteristics by themselves are not enough to distinguish the two classes of objects (Caswell 1999). The situation for $\mathrm{SiO}$ maser sources is also complex. For example, there are three $\mathrm{SiO}$ maser sources in molecular clouds (Hasegawa 1985; Morita et al. 1992) that are likely to be young stellar objects. There are also peculiar cases, such as the $\mathrm{SiO}$ maser source IRAS $19312+1950$ is an evolved star in a dark cloud (Nakashima et al. 2004; Deguchi et al. 2004c), and V838 Mon is an M supergiant with $\mathrm{SiO}$ emission which emerged after nova eruption (Deguchi et al. 2005b; Tylenda \& Soker 2006).

In some cases, the imaging and identification of the central star in NIR bands can greatly facilitate the determination of the nature of these objects (Lewis et al. 2004; Deguchi et al. 2005a). Young, low-mass stars are usually born in a star cluster, but evolved stars, especially AGB/postAGB stars with the ages of Giga years, are observed as a single object (see $\S 3$ of Deguchi et al. 2004c). However, for the case of supergiants, they are frequently a member of stellar associations (Humphreys 1970), suggesting they are on their way towards leaving the originating star clusters. Deep infrared imaging can help find the surrounding low-mass stars.

The recently performed Galactic Legacy Infrared Mid-Plane Survey Extraordinaire (GLIMPSE) of the Spitzer Space Telescope (SST) has provided unprecedented deep near-infrared images of the Galactic plane. Because of the high angular resolution $1.2^{\prime \prime}$ per pixel) of the GLIMPSE observa- tions, we have the opportunity to obtain an accurate identification of the infrared/maser sources. In this paper, we report the identification of 41 infrared sources from the GLIMPSE data, as well as $\mathrm{SiO}$ or $\mathrm{H}_{2} \mathrm{O}$ maser observations of these sources with the Nobeyama 45-m telescope.

\section{Observations}

\subsection{Source selection}

We selected 41 sources from the Midcourse Space Experiment (MSX) Galactic plane survey that are found to have no (or dubious) nearinfrared counterparts in the 2MASS sky survey (Skrutskie et al. 2000). Half of these (22 objects) are $\mathrm{OH} / \mathrm{IR}$ objects listed by Sevenster et al. (2001), te Lintel-Hekkert et al. (1991), Eder et al. (1988), etc. The positional accuracy of the MSX positions is about a few arcseconds (Egan et al. 1999), comparable to the positional accuracies of $\mathrm{OH}$ masers measured with the Very Large Array (VLA) [see figure 9 of Deguchi et al. (2002) for comparison]. Most of these objects have reasonably red mid-IR colors with $C_{C E}\left[\equiv \log \left(F_{E} / F_{C}\right)\right]$ between 0 and 0.5 , where $F_{C}$ and $F_{E}$ are the MSX band $\mathrm{C}(12 \mu \mathrm{m})$ and band $\mathrm{E}(21 \mu \mathrm{m})$ flux densities, respectively.

In addition to the MSX sources, we have included a number of medium-bright and red IRAS sources without 2MASS counterparts for which no maser line search was made. These are IRAS 18030-1858, 18151-1713, 18241-1010, 18298-0904, 18424-0509, 18444-0359, 19011+0638, $19025+0702,19087+1006,19112+1220,19114+0920$, $19180+1230,19254+1724,19266+1815,19275+1720$, G014.2758-00.1602, G027.8433-00.1060. The MSX colors of these objects are not much different from those of the above $\mathrm{OH} / \mathrm{IR}$ sources without NIR counterparts. The entire sample we chose for identification in the Spitzer/GLIMPSE survey is listed in table 1, giving the source name, MSX $(6 \mathrm{C})$ name, right ascension and declination (J2000) of the MSX source, flux density in the MSX C $(12 \mu \mathrm{m})$ band, the MSX colors $\left[C_{A C}=\log \left(F_{C} / F_{A}\right)\right.$, and $\left.C_{C E}=\log \left(F_{E} / F_{C}\right)\right]$, status of detections in $\mathrm{SiO}, \mathrm{H}_{2} \mathrm{O}$, and $\mathrm{OH}$ masers, and a conventional $\mathrm{OH}$ object name. 


\subsection{Spitzer/GLIMPSE Identification}

The GLIMPSE survey was made with SST between March and November, 2004 using the Infrared Array Camera (IRAC) (Indebetouw et al. 2004). The survey covered the area of $|l|=10-65^{\circ}$ over latitudes $|b|<1^{\circ}$ toward the inner Galactic disk excluding the Galactic center. Simultaneous $5.2^{\prime} \times 5.2^{\prime}$ images at 3.6, 4.5, 5.8 and $8 \mu \mathrm{m}$ were obtained at a spatial resolution of $\sim 1.2^{\prime \prime} \times 1.2^{\prime \prime}$ per pixel.

The color-composite images (from the 3.6, 4.5 and $5.8 \mu \mathrm{m}$ band images) toward the 41 selected objects are shown in Figure 1. The counterparts for all sources in our sample were found; they are all very red and near the center of the images. Because all of these objects are bright in $8 \mu \mathrm{m}$, the identifications in other bands were made quite easily. The color images were created with special software developed at the Space Astronomy Laboratory of the University of Calgary. The flux densities were derived from the GLIMPSE archives; the aperture size used for photometry was typically a few to 12 arcseconds, depending on the image size of the star. The estimated error of the photometry is dependent on the background noise levels, and are typically $<2 \%$ for the objects brighter than 1, 3, 7, 16 mJy at the 3.6, 4.5 and $5.8 \mu \mathrm{m}$ bands respectively. The flux densities are given in columns $4-7$ in table 2 . The color indices, [3.6] - [5.8] and [3.6] - [12], are also listed in the 8th and 9th columns in table 2. Here, [3.6], [5.8], and [12] are the magnitudes in the GLIMPSE 3.6 and and $5.8 \mu \mathrm{m}$, and MSX $12 \mu \mathrm{m}$ bands, respectively, and the flux densities at 0th magnitude are 280.9, 115.6, and 26.4 Jy in these bands, respectively. The interstellar extinction toward each object is given in the 10th column of table 2 and it is estimated by the formula based on the fullsky $100 \mu \mathrm{m}$ composite map of COBE/DIRBE and IRAS maps (Schlegel et al. 1998) 1]

After getting accurate GLIMPSE positions (the 2nd and 3rd columns in table 2), we again checked the 2MASS images and found the red counterparts corresponding to the positions of 4 sources; 18286-0959 (J18312292-0957194), 18298-0904 (J18323700-0902301), 19275+1902 $(J 19294645+1709013)$, and $19266+1815(J 19285303$ $+1821228)$. These counterparts are very faint in the $\mathrm{K}$-band ( $K=12.6-14.7)$, making previous identifications uncertain. Their positions coincide with those of the GLIMPSE objects within $0.5^{\prime \prime}$. Jiménez-Esteban et al. (2006) listed NIR counterparts for 5 objects in the present sample: 18182-1504, 18460-0254, 18488-0107, $19087+1006$, and $19254+1631$. Because of the faintness of the objects $(K>13)$, their identifications were again doubtful. We have checked the GLIMPSE positions of these objects and found that 18182-1504, 18460-0254, and 19254+1631 were correctly identified, but 18488-0107 and 19087+1006 were misidentified; the GLIMPSE objects are located $5^{\prime \prime} \mathrm{NE}$ and $66^{\prime \prime} \mathrm{S}$ of the 2MASS stars ( $K=13.05$ and 14.10) given by Jiménez-Esteban et al. (2006).

In addition to the above selected objects without 2MASS counterparts, we checked the GLIMPSE images of about $200 \mathrm{SiO}$ maser sources within the area of $l=10-60^{\circ}$, and $|b|<1^{\circ}$ where the near-infrared identifications are already available (Deguchi et al. 1998, 2001, 2002). The GLIMPSE images mostly exhibit a single bright object at the center of the field, except for IRAS $19027+0517$, which shows accompanying nebulosity (Fig. 6). Because this object has a NIR counterpart in 2MASS images and seems to be intrinsically different from the objects in our sample, we gave a discussion of this object separately in Appendix A.

We wish to make a special reference to IRAS 18450-0148 (W43A), which is known as a water maser fountain with collimated outflows (Imai et al. 2002). The detection of $\mathrm{SiO}$ maser emission in this object (Nakashima \& Deguchi $2003 \mathrm{~b})$ suggests that this is an evolved star. The near-infrared counterpart of this interesting object is identified here for the first time.

\section{3. $\mathrm{SiO}$ and $\mathrm{H}_{2} \mathrm{O}$ maser observations at Nobeyama}

Because a majority of objects in our sample have not been searched for $\mathrm{SiO}$ or $\mathrm{H}_{2} \mathrm{O}$ maser emissions before, we observed the objects in $\mathrm{SiO}$ $J=1-0 v=1$ and 2 and $\mathrm{H}_{2} \mathrm{O} 6_{16}-5_{23}$ maser lines with the 45-m telescope at Nobeyama during April 10-23, 2006. A cooled HEMT receiver (H40) and an accousto-optical spectrometer array were used for the $43 \mathrm{GHz}$ observations. The receiver system temperature was about $200 \mathrm{~K}$ and the effective 
velocity resolution is $0.3 \mathrm{~km} \mathrm{~s}^{-1}$. The half-power beam width (HPBW) at $43 \mathrm{GHz}$ was $\sim 40^{\prime \prime}$ and the conversion factor of the antenna temperature to the flux density was $\sim 2.9 \mathrm{Jy} \mathrm{K}^{-1}$. In addition to the $\mathrm{SiO}$ maser observations, we made a 22.235 $\mathrm{GHz} \mathrm{H}_{2} \mathrm{O}$ maser observation on April 20 and 23, when weather was unfavorable for $43 \mathrm{GHz}$ observations. We used a cooled HEMT receiver (H22) and the same accousto-optical spectrometer array (with an effective velocity resolution of $0.6 \mathrm{~km}$ $\mathrm{s}^{-1}$ ). The conversion factor of the antenna temperature to the flux density was $\sim 2.8 \mathrm{Jy} \mathrm{K}^{-1}$ at $22 \mathrm{GHz}$. Because the $\mathrm{H} 40$ and $\mathrm{H} 22$ receivers have a $2 \mathrm{GHz}$ band width, we configured the AOS-H spectrometer array to have the spectra of the $\mathrm{SiO}$ $J=1-0 v=0(43.423 \mathrm{GHz})$, and $v=3(42.519$ $\mathrm{GHz}),{ }^{29} \mathrm{SiO} J=1-0, \mathrm{v}=0(42.880 \mathrm{GHz})$, and H53 $\alpha$ (42.952 GHz) lines simultaneously in the $\mathrm{H} 40$ receiver, and $\mathrm{NH}_{3} J_{K}=1_{1}-1_{1}, 2_{2}-2_{2}$, and $3_{3}-3_{3}$ lines $(23.694,23.722,23.870 \mathrm{GHz}$, respectively) simultaneously in the $\mathrm{H} 22$ receiver. The rms noise temperatures for these additional lines are similar to the noise levels of the $\mathrm{SiO}$ and $\mathrm{H}_{2} \mathrm{O}$ lines and therefore are not repeated in tables 3 and 4. No detections in these additional lines were obtained except for 18182-1504. Further details of observations using the NRO 45-m telescope are described elsewhere (e.g., Nakashima \& Deguchi 2003a).

Among the 31 objects observed, 8 were detected in $\mathrm{SiO}$ and their spectra are shown in Fig. 2. In all cases, the $v=2$ maser line is stronger than the $v=$ 1 maser line. Water masers in the $6_{16}-5_{23}$ transition were detected in two sources (18286-0959 and 18460-0151) and their spectra are shown in Fig. 3. It is notable that both of the detected objects exhibit a wide velocity range in the $\mathrm{H}_{2} \mathrm{O}$ maser emission spectra, which resemble the $\mathrm{H}_{2} \mathrm{O}$ spectra of "water fountains" (Morris et al. 2003).

Because some of our sample objects might be associated with young stellar objects in molecular clouds, we also made simultaneous observations of the $\mathrm{HCO}^{+} J=1-0$ line at $89.189 \mathrm{GHz}$ and the $\mathrm{SiO} J=2-1 v=1$ line. The non-detection of the $\mathrm{HCO}^{+}$line effectively excludes the possibility of these water maser sources being associated with young stellar objects.

\section{Discussion}

\subsection{Two-color diagram}

The selected objects have extremely thick circumstellar envelopes. In addition, they suffer from a large interstellar reddening in the Galactic plane even at NIR to MIR wavelengths. Therefore, it is hard to separate the circumstellar from the interstellar reddening for these objects because of the uncertainty of the interstellar extinction correction 2 We discuss here quantities of the sampled objects by introducing the color indices, [3.6]- [5.6] and $[3.6]-[12]$, which vary relatively mildly with the large extinction. We apply a wavelengthdependent extinction derived from the GLIMPSE survey which was obtained from the color excess of stars at $l=42^{\circ}$ and $284^{\circ}$ (Indebetouw et al. 2005). We compare physical quantities of the sample objects with those of the proto-typical OH/IR star with $\mathrm{SiO}$ masers, $\mathrm{OH} 127.8+0.0$, which is believed to be at the final stage of evolution on the AGB. Physical parameters of this star are relatively well known (Suh \& Kim 2002), with the optical depth of the envelope being about 10-15 at $9.8 \mu \mathrm{m}$. This bright $\mathrm{OH} / \mathrm{IR} \operatorname{star}\left(F_{C}=184\right.$ Jy) has $[3.6]-[5.8]=3.46$ and $[3.6]-[12]=5.0$. Because of the position $\left(l \sim 128^{\circ}\right)$, the interstellar extinction at the NIR wavelengths is small for this object $\left(A_{K}=0.64\right)$. If this object were behind a dust cloud with a heavier extinction of $A_{K}>7.6$, it would be undetectable on the 2MASS image $(K>13.7)$. The spherically symmetric model for $\mathrm{OH} 127.8+0.0$ (Kemper et al. 2002) gave a mass loss rate of $7 \times 10^{-5} \mathrm{M}_{\odot} \mathrm{yr}^{-1}$. The same model gives a total mass of the envelope of $0.2 \mathrm{M}_{\odot}$ (if integrated to the outer radius of the dust shell, $1.3 \times 10^{17} \mathrm{~cm}$, as used by Kemper et al. (2002)).

Figure 4 shows a two-color diagram, [3.6] - [12] versus [3.6] - [5.8], for the sampled objects, where $\mathrm{OH} 128.7+0.0$ occupies the position indicated by the square in this diagram. The interstellar reddening moves the position of the star to the upper right with an inclination of about unity because the extinctions at 5.8 and $12 \mu \mathrm{m}$ are comparable (Table 1 of Mathis 1990). Selected objects in this diagram distribute from the lower-left to the up-

2 As noted in Section 2.2, we used a formula given by Schlegel et al. (1998), which gives almost the maximum value of interstellar extinction due to thick dust clouds toward each source. 
per right with a steeper slope $(\sim 1.71 \pm 0.19)$ than the slope of the interstellar reddening line $(\lesssim 1)$. They appear in a very wide range of color indices, over 3 in [3.6] - [5.8] (corresponding to $A_{K}=20$ ), which is too large to be considered an effect of interstellar reddening. These facts suggest that the extremely red colors of these objects cannot be explained solely by the interstellar reddening applied to the $\mathrm{OH} 127.8+0.0$ type star, but they are more or less intrinsic to these objects.

The steepness of the distribution seems to be understandable by the model that the $12 \mu \mathrm{m}$ flux is a re-emission of absorbed NIR light by dust grains near the central star. If MIR re-emission does not occur (as interstellar reddening), the star should move on the line indicated by the dotted arrow. $\mathrm{SiO}$ detections (filled circles) also appear in a wide range of color indices in figure 4 , but the $\mathrm{SiO}$ sources distribute with a steeper inclination $(\sim 1.98 \pm 0.35)$ than the no-SiO subsample $(\sim 1.62 \pm 0.21)$, indicating that the re-emission effect at $12 \mu \mathrm{m}$ is stronger in the $\mathrm{SiO}$ maser sources than the non-SiO emitting objects. The $\mathrm{SiO}$ maser is an indicator of active mass loss near the central star $\left(\sim 10^{14} \mathrm{~cm}\right)$, suggesting the presence of thick dust re-emitting the stellar radiation more at MIR wavelengths than the objects without $\mathrm{SiO}$ masers.

The above findings strongly suggest that these objects have an excessively large optical depth of circumstellar dust, which is much larger than that of the circumstellar dust of $\mathrm{OH} 127.8+0.0$. The order of magnitudes of the excess material can be estimated from [3.6] $-[12]$. We use the relation between $[3.6]-[12]$ and the mass loss rate calculated by Kemper et al. (2002). Applying the interstellar reddening corrections to [3.6] - [12], we computed the mass loss rate of the envelope from figure 6 of Kemper et al. (2002), and obtained the excess factor $\left(f_{c}\right)$ of the circumstellar matter (relative to that of the $\mathrm{OH} 127.8+0.0$ envelope), which is listed in the last column of table 2 . They exceed the optical depth of the $\mathrm{OH} 127.8+0.0$ envelope by a factor of a few up to 80 . Uncertainty of the interstellar extinction correction of about $30 \%$ makes a shift of [3.6] - [12] up to 0.7 at most, making the uncertainty of the excess factor to be within a factor of 2 even for the worst case.

The total envelope mass for each sampled object must exceed by a similar factor, i.e. these objects might have envelope masses of $0.4-20 M_{\odot}$ if simply integrated until the outer radius of $\sim 10^{17}$ $\mathrm{cm}$. The total mass of the envelope is proportional to the outer-boundary radius for the assumed density distribution of a constant-mass-loss model $\left(\rho \propto r^{-2}\right)$, whereas the optical depth is rather sensitive to the inner-bounday radius of the dust envelope. Because the color index, [3.6]-[12], which was used here for evaluation of the excess factor, does not give useful restrictions on the outer boundary radius in the Kemper et al's modeling (it is rather restricted by IRAS 60 or 100 $\mu \mathrm{m}$ flux density), the total mass of the thick matter estimated here has large ambiguity. Because, without precise modeling, it is uncertain as to how far from the central star the extra material in the envelope is located, we only use the excess factor $f_{c}$ in this paper, which characterizes the envelope of these objects.

The total envelope mass for each sampled object can be reduced, in some degree, by introducing a non-spherical distribution of the dust envelope, e.g. a torus or disk structure. However, even a wide opening angle of $120^{\circ}$ for cavity cones can reduce the mass by $50 \%$ compared with that of the spherical distribution with the same radial density profile. As discussed in the later sections, highvelocity components of water maser emission may suggest the presence of the accretion disk which creates collimated jets. In such a case, the scattered light through the pole of the torus should be observable, although it is hard to detect the scattered light for these objects because of the large interstellar extinction in the $K$ band.

\subsection{Characteristic of $\mathrm{SiO}$ maser emission}

It is striking that only the $\mathrm{SiO} J=1-0 v=2$ line was detected in 6 out of $8 \mathrm{SiO}$ detected sources. Even in the remaining two sources the intensity of the $J=1-0 v=2$ line was considerably stronger than that of the $J=1-0 v=1$ line. The trend, i.e. the increase of the $v=2 / 1$ line intensity ratio with the infrared color $\left[C_{12}=\right.$ $\log \left(F_{25} / F_{12}\right)$, where $F_{12}$ and $F_{25}$ are IRAS 12 and $25 \mu \mathrm{m}$ flux density], was clearly first demonstrated by Nakashima \& Deguchi (2003b). For the present sample of $\mathrm{SiO}$ detected sources, the MSX color, $C_{C E}=\log \left(F_{21} / F_{12}\right)$, is between 0.10 and 0.34 , which fits well with the above trend. All of the $\mathrm{SiO}$ detected sources were previously observed by the $\mathrm{OH} 1612 \mathrm{MHz}$ line and were all 
detected except for 18241-1010.

Figure 4 indicates that, for the sampled objects, $\mathrm{SiO}$ maser detection rates do not seem to correlate with the colors, [3.5] - [5.8], or [3.6] - [12]. The flat detection rate seems to suggest that these stars are still in a mass-losing stage, at the final transient stage of the AGB to planetary-nebula phase. They are not at a later stage of the post-AGB phase when $\mathrm{SiO}$ masers should disappear.

\subsection{New $\mathrm{H}_{2} \mathrm{O}$ sources with wide spread emission}

Two $\mathrm{H}_{2} \mathrm{O}$ maser sources, 18286-0959 and 18460-0151 (Fig. 3) have a very wide velocity range $\left(\gtrsim 200 \mathrm{~km} \mathrm{~s}^{-1}\right)$ in their maser emission spectra. Although the rich $\mathrm{H}_{2} \mathrm{O}$ maser emission spectrum in IRAS 18286-0959 (Fig. 3 left) resembles the water maser spectra associated with compact H II regions (for example, Kurtz \& Hofner 2002), the nondetections of $\mathrm{HCO}^{+}, \mathrm{H} 53 \alpha$, and $\mathrm{NH}_{3}$ lines toward these sources (the present work: table 3 ) suggest that they are not associated with dense molecular clouds (or compact H II regions), and the rich $\mathrm{H}_{2} \mathrm{O}$ emission could be the result of a very irregular velocity field in the envelope. The fact that these sources are point-like with no associated nebulosity in their GLIMPSE images suggests that they are stellar objects.

Similar wide spread water maser spectra were found in the class of "water fountain" sources. There are four known water fountain sources [16342-3814, OH 12.8+0.9 (18139-1816), W43A (18450-0148), $\quad 19134+2131$ (Likkel \& Morris 1988; Boboltz et al. 2005; Imai et al. 2004, 2005) with a few more probable cases (Gomez et al. 1994; Deacon et al. 2001). All of these objects exhibit the $\mathrm{H}_{2} \mathrm{O}$ maser components in a velocity range that exceed the $\mathrm{OH}$ velocity range. The $\mathrm{H}_{2} \mathrm{O}$ maser components are spatially more extended than the $\mathrm{OH}$ maser components (Imai et al. 2004; Boboltz et al. 2005). Among these "water fountain sources", 16342-3814 had the highest velocity separation of $\sim 258 \mathrm{~km} \mathrm{~s}^{-1}$. Our object 18460-0151 definitely belongs to the class of water fountain sources from the similarity of the emission characteristic, and it has a recordhigh separation of $292 \mathrm{~km} \mathrm{~s}^{-1}$ between emission components.

Both new objects, 18286-0959 and 18460-0151, are relatively bright IRAS sources (25 and $20 \mathrm{Jy}$ at $12 \mu \mathrm{m}$ respectively); the former has a $2 \mathrm{MASS}$ counterpart, $J 18312292-0957194$, with $K$ magnitude of 12.67 and $H-K=0.89$. They have a rising mid-IR spectrum toward longer wavelengths, indicating the presence of a thick dust envelope. From the radial velocities, we estimate kinematic distances of 18286-0959 and 18460-0151 as 3.1 and $6.8 \mathrm{kpc}$, respectively, and luminosities as $8.7 \times 10^{3}$ and $4.2 \times 10^{4} L_{\odot}$ for these distances.

If these objects have a thick dust torus (as in the unified model of the Type I and II active galactic nuclei), optically observable objects (as IRAS 16342-3814; Sahai et al. 1999) must be seen from the polar-axis direction of the dust torus and unseen from equatorial directions. If these highvelocity features are a part of the polar jet, which is created by the accretion disk, the higher velocity objects should have bluer colors. This hypothesis seems applicable for 18286-0959 (because of the identified NIR counterpart with $K=12.67$ ), but not for 18460-0151 (with no NIR counterpart). Regarding 18460-0151, which has the highest velocity separation among the water fountains, the large distance $(\sim 6.8 \mathrm{kpc})$ and the strong interstellar extinction hide this object behind molecular clouds. Applying the large interstellar extinction correction of $A_{K}=8.2$, we infer the extinction corrected $K$ magnitude to be fainter than 5.8. It is slightly fainter than the extinction-corrected 3.6 $\mu \mathrm{m}$ magnitude $([3.6] \sim 5.5)$, suggesting that the scattered light is not seen from this object.

\subsection{Envelopes of extremely red $\mathrm{OH} / \mathrm{IR}$ stars}

The central stars of extremely red $\mathrm{OH} / \mathrm{IR}$ objects with low expansion velocity $\left(V_{\text {exp }}<15\right.$ $\mathrm{km} \mathrm{s}^{-1}$ ) have been considered to be relatively low-initial-mass post-AGB stars compared with those of normal-color sources (Sevenster et al. 2002). In fact, the red ("RI") group sources of Sevenster et al. (2002) involved two objects in the present sample: IRAS 18135-1456 and $18596+0315$, which have the expansion velocities, 14.8 and $13.6 \mathrm{~km} \mathrm{~s}^{-1}$, respectively. Regarding expansion velocites derived from $\mathrm{OH}$ peaks, two water fountains, 18450-0148 (W43A) and 18460-0151, are low expansion-velocity (lowermass) sources $\left(V_{\text {exp }}=6\right.$ and $\left.11 \mathrm{~km} \mathrm{~s}^{-1}\right)$.

Figure 5 shows a plot of the excess factor of the 
envelope versus expansion velocity for $\mathrm{OH} 1612$ $\mathrm{MHz}$ doubly-peaked objects in the present sample. Note that the excess factor was derived from [3.6] - [12] (relative to $\mathrm{OH} 127.8+0.0$; see section 3.1). It indicates that the excess factor does not correlate strongly with the expansion velocity of the envelope, and hence the initial mass of the central star.

Figure 5 also indicates that objects with small expansion velocity $\left(V_{\text {exp }}<15 \mathrm{~km} \mathrm{~s}^{-1}\right)$ seem to have lower $\mathrm{SiO}$ detection rates than the larger expansion-velocity objects $\left(V_{\exp }>15 \mathrm{~km} \mathrm{~s}^{-1}\right)$. This indicates that the lower-mass stars with small expansion velocities are at the phase unfavorable for making strong $\mathrm{SiO}$ masers, e.g. dissociating $\mathrm{SiO}$ molecules by hot central star radiation, and/or terminating mass loss from the central star. This is consistent with the Sevenster et al. (2002)'s finding that the red objects with small expansion velocites are stars in the early postAGB phases with nearly zero mass loss; the extreme high-velocity flow develops at this phase and $\mathrm{SiO}$ masers gradually disappear. We infer that the red stars with large expansion velocites are AGB stars still in an active mass-losing stage because of the presence of $\mathrm{SiO}$ masers. However both groups of stars, which are investigated in this paper, are dressed by excessively large amounts of circumstellar matter irrespective of the mass of the central star.

The radii of the $\mathrm{OH}$ emitting regions were found to be $3-16 \times 10^{16} \mathrm{~cm}$ for some of the selected objects $(\mathrm{OH} 21.5+0.5,30.1-0.7$, and 32.0-0.5) with the $\mathrm{OH}$ phase-lag/angular-size measurements (Herman et al. 1985). Because the $\mathrm{H}_{2} \mathrm{O}$ masing region of water fountains is extended more than the $\mathrm{OH}$ emission region (Imai et al. 2002, for W43A), the thick material must be extended out of the $\mathrm{OH}$ masing region. There is evidence that $\mathrm{H}_{2} \mathrm{O}$ maser outflow strikes the dense material which is located outside of the $\mathrm{OH}$ masing region. Therefore, it is likely that the excess material found in section 3.1 is extended to considerably outer parts of the envelope, i.e. outside of the $\mathrm{OH}$ masing region. The $\mathrm{H}_{2}$ number density of the $\mathrm{OH}$ masing region is known to be $\sim 10^{3}-$ $10^{4} \mathrm{~cm}^{-3}$ (for example, Netzer \& Knapp 1987), and the density of the $\mathrm{H}_{2} \mathrm{O}$ masing region must be, by several orders of magnitude, higher $\left(\sim 10^{9}\right.$ $\mathrm{cm}^{-3}$ ). Thus, we cannot deny the possibility that the thick layer is extended at the radius of more than $3 \times 10^{16} \mathrm{~cm}$, though such a layer could be clumpy and dense, depending on the model. Envelope masses of some $\mathrm{OH} / \mathrm{IR}$ stars [e.g., IRAS 18450-0148 (W43A)], exceed $4 M_{\odot}$ even for the outer radius of $3 \times 10^{16} \mathrm{~cm}$. Though the envelope mass can vary by a factor of a few, depending on the uncertainty of interstellar extinction and nonspherical distribution of the thick layer as noted before, it is difficult to lower the envelope mass less than $1 M_{\odot}$ for several of the thickest objects of the sample.

A number of numerical modelings of thick circumstellar envelopes in the outgoing AGB phase have been made (for example, van Hoof et al. 1997; Lorenz-Martins \& de Araujo 1997). David \& Papoular (1992) and Suh \& Jones (1997) examined superwind models to explain far IR spectra of $\mathrm{OH} / \mathrm{IR}$ objects accompanying deep silicate absorptions. One of their models, which has a dense shell between radii $2 \times 10^{16}$ and $4 \times 10^{16} \mathrm{~cm}$ in addition to a normal continuous flow (Suh \& Jones 1997), successfully fits the computed to the observed spectral energy distribution of IRAS 18257-1000 $(\mathrm{OH} 21.5+0.5)$, which has a relatively mild excess factor (1.4) in our list (table 2). The large IRAS $60 \mu \mathrm{m}$ flux densities of most of the other objects (though some of them might be contaminated from nearby clouds) entail for the much denser outer shell in these models, which inevitably results in a large total envelope mass of more than a few $M_{\odot}$ (David \& Papoular 1992); the superwind models artificially restrict the outer radius of the thick material to keep the enclosed mass smaller. Although the total mass of the outer envelope could in principle be observationally constrained by CO $J=1-0$ intensities or IRAS $60 / 100 \mu \mathrm{m}$ flux densities, it is hard to estimate for these objects because of contamination by radiation from surrounding clouds.

Though all of these modelings of very red $\mathrm{OH} / \mathrm{IR}$ objects assume a superwind with a mass loss rate of $\sim 10^{-4} M_{\odot} \mathrm{yr}^{-1}$ in a duration of about a few hundred years, the origin of the thick material is not necessarily restricted to the superwinds which occur at the end of the AGB phase. Because no correlation appears between the excess factor and the expansion velocity, the thick material does not seem to be related with the central star masses. The excess mate- 
rial at the outer envelope of these objects can be a source of a shock front of the extremely high-velocity outflow created at the early postAGB phase of stars, that is observed as water fountains. This may be due to gas ejection by binary-star interactions (Nordhaus \& Blackman 2006). Men'shchikov et al. (2002) found that the massive dust torus of the Red Rectangle has $M \sim 1.2 M_{\odot}$, which was formed in the ejection of a common envelope after Roche lobe overflow. Similarly, stellar merging (for example, Bally \& Zinnecker 2005; Tylenda \& Soker 2006) may create such massive circumstellar material. Alternatively these stars may simply be in a dense dust cloud, as found in IRAS $19312+1950$ (Deguchi et al. 2004a). We cannot deny such a possibility for some of these sources (but not all of them) because they are seen toward thick dust clouds. We infer here that massive circumstellar matter of the thickest $\mathrm{OH} / \mathrm{IR}$ stars in the present sample can be formed by one of the aforementioned mechanisms.

\section{Conclusion}

$\mathrm{SiO}$ maser and $\mathrm{OH} / \mathrm{IR}$ objects with no 2MASS counterparts were identified in the GLIMPSE 3.6/4.5/5.8/8.0 $\mu \mathrm{m}$ images with a spatial resolution of $1.2^{\prime \prime}$. Searches for $\mathrm{SiO}$ and $\mathrm{H}_{2} \mathrm{O}$ masers lead to 10 new detections, each of which show characteristic maser spectra of the stronger $\mathrm{SiO}$ $J=1-0 v=2$ to $v=1$ line or wide spread $\mathrm{H}_{2} \mathrm{O}$ maser emission. It turned out that the dust envelopes of these objects are exceptionally thick, though they suffer from a large interstellar extinction. This suggests that a considerable mass of materials toward the objects must exist in the envelope. The $\mathrm{SiO}$ detection rate was uncorrelated with the mass of the envelope, but the lower detection rate was obtained for the lower-expansion velocity subsample of the $\mathrm{OH} / \mathrm{IR}$ stars. The latter suggests that the higher-expansion velocity objects are AGB stars and lower-expansion velocity objects are more evloved stars such as post-AGB stars. The derived envelope mass is not correlated with this sequence of stellar evolution. From these observations, we believe that the thick material in some of these objects cannot be ascribed to the AGB-phase wind of the central star.
We thank Ed Churchwell and the GLIMPSE team for their help in the retrieval and processing of the survey data. We also thank the anonymous referee for useful comments for clarifing the content. This research made use of the SIMBAD and VizieR databases operated at CDS, Strasbourg, France, and as well as use of data products from Two Micron All Sky Survey, which is a joint project of the University of Massachusetts and Infrared Processing and Analysis Center/California Institute of Technology, funded by the National Aeronautics and Space Administration and $\mathrm{Na}-$ tional Science foundation, and from the Midcourse Space Experiment at NASA/ IPAC Infrared Science Archive, which is operated by the Jet Propulsion Laboratory, California Institute of Technology, under contract with the National Aeronautics and Space Administration. This work is supported in part by a grant to SK from the Natural Sciences and Engineering Research Council of Canada. 


\section{A. IRAS $19027+0517$}

The Glimpse images of the $\mathrm{SiO}$ maser source, IRAS 19027+0517, exhibit conspicuous nebulosity around the central star at 5.8 and $8.0 \mu \mathrm{m}$ (Fig. 6). The nebula is lopsided to the central star and extended to the north. The surface brightness of the nebula seems to increase toward the central star, which suggests a physical association of this nebula to the central star. Among $200 \mathrm{SiO}$ sources investigated, only this object has an extended nebulosity. The 2MASS images show a medium bright central star $(K=8.2)$, but do not exhibit any extended features. The nebulous feature is considered to be due to the PAH emission with sharp spectral features at 6.2 and $7.8 \mu \mathrm{m}$ (Verstraete et al. 2001). It is quite unusual that the O-rich central star in the AGB phase (with $\mathrm{SiO}$ masers) accompanies such extended PAH nebulosity. Therefore, we particularly investigated this object more with various molecular lines with the NRO $45 \mathrm{~m}$ telescope. In addition to the previous detections of the $\mathrm{SiO} J=1-0 v=1$ and 2 lines (Deguchi et al. 2004b) , we found emissions of the $\mathrm{SiO} J=1-0 v=0$ and 3 , and $J=2-1 v=1$ lines; the spectra are shown in figure 7 . Furthermore, we made $45^{\prime \prime}$-grid 5-point mapping observations by $\mathrm{CO}, \mathrm{HCN}$ and $\mathrm{HCO}^{+} J=1-0$ lines, but no emission associated with the central star was detected, except for the fore/background clouds with large velocity separations (see figure 8). The observational results are summarized in table 5. Therefore, we conclude that most of the molecules are dissociated in the nebula toward IRAS 19027+0517, an O-rich AGB star, by UV radiation from a closeby UV source creating the lopsided nebulosity. 


\section{REFERENCES}

Allen, D. A., Hyland, A. R., Longmore, A. J., Caswell, J. L., Goss, W. M., \& Haynes, R. F. 1977, ApJ, 217, 108

Boboltz, D. A. \& Marvel, K. B. 2005, ApJ, 627, L45

Bally, J. \& Zinnecker, H. 2005, AJ, 129, 2281

Caswell, J. L. 1999, MNRAS, 308, 683

Chengalur, J. N., Lewis, B. M., Eder, J., \& Terzian, Y. 1993, ApJS, 89, 189

David, P. \& Papoular, R. 1992, A\&A, 265, 195

Deacon, R. M., Chapman, J. M., Green, A. J., \& Sevenster, M. N. 2006, submitted to ApJ

Deguchi, S., Fujii, T., Matsumoto, S. Nakashima, J., \& Wood, P. 2001, PASJ, 53, 293

Deguchi, S., Fujii, T., Nakashima, J., \& Wood, P. R., 2002, PASJ, 54, 719

Deguchi, S., et al. 2004a, PASJ, 56, 261

Deguchi, S. et al. 2004b, PASJ, 56, 765

Deguchi S., Matsumoto S., \& Wood P. R. 1998, PASJ, 50, 597

Deguchi, S., Nakashima, J., Takano, S. 2004, PASJ, 56, 1083

Deguchi, S., Nakashima, J., Miyata, T., \& Ita, Y. 2005, PASJ, 57, 933

Deguchi, S., Matsunaga, N., Fukushi, H. 2005, PASJ, 57, L25

Eder, J., Lewis, B. M. \& Terzian, Y. 1988, ApJS, 66,183

Engels, D., Schmid-Burgk, J., \& Walmsley, C. M. 1986, A\&A, 167, 129

Engels, D. \& Lweis, B. M. 1996, A\&AS, 116, 117

Egan M. P., et al. 1999, Air Force Research Laboratory Technical Report No. AFRL-VS-TR-1999-1522 (available at IPAC, 〈http://irsa.ipac.caltech.edu/applications/MSX

Gomez, Y., Moran, J. M. \& Rodriguez, L. F. 1990, RMxAA, 20, 55
Gomez, Y., Rodriguez, L. F., Contreras, M. E., \& Moran, J. M., 1994, RMxAA, 28, 97

Habing, H. J. 1996, A\&AR, 7, 97

Hasegawa, T. et al. 1985, in Masers, Molecules and Mass Outflows in Star Forming Regions, ed. A. D. Haschick (Aystack Observatory, Westford), p. 275

Herman, J., Baud, B., Habing, H. J., \& Winnberg, A 1985, A\&A143, 122H

Humphreys, R. M. 1970, AJ, 75, 602

Imai, H. Obara, K., Diamond, P. J., Omodaka, T., \& Sasao, T. 2002, Nature, 417, 829

Imai, H., Morris, M., Sahai, R., Hachisuka, K., \& Azzollini F. J. R. 2004, A\&A, 420, 265

Imai, H. Nakashima, J., Diamond, P.J., Miyazaki, A. \& Deguchi, S. 2005, ApJ, 622, L125

Indebetouw, R., Benjamin, R. A., \& Churchwell, E. 2004, ASPC, 317, 109 "Milky Way Surveys: The Structure and Evolution of Our Galaxy" ed. by D. Clemens, R. Shah, \& T. Brainerd

Indebetouw, R. et al. 2005, ApJ, 619, 931

Izumiura, H. et al. ApJS, 125, 257

Jiménez-Esteban, F. M., Agudo-Mérida, L., Engels, D., García-Lario, P. 2005, A\&A, 431, 779

Jiménez-Esteban F. M., García-Lario, P., Engels, D., \& Perea Calderón, J. V. 2006, A\&A, 446, 773

Kemper, F., De Koter, A., Waters L. B. F. M., Bouwman, J., Tielens, A. G. G. M. 2002, A\&A, 384,585

Kurtz, S. \& Hofner, P. 2005, AJ, 130, 711

Kwok, S. 1976, JRASC, 70, 49

Kwok, S. in The origin and evolution of Planetary nebulae (Cambridge: Cambridge Univ. Press)

Kwok, S., Volk, K., and Bidelman, W.P. 1997, ApJS, 112, 557

Lewis, B. M., Eder, J., \& Terzian, Y. 1987, AJ, 94,1025 
Lewis, B. M., Kopon, D. A., \& Terzian, Y. 2004, AJ, 127, 501

Likkel, L., \& Morris, M., 1988, ApJ, 329, 914

Likkel, L., Morris, M., \& Maddalena, R. J. 1992, A\&A, 256, 581

Lorenz-Martins, S. \& de Araujo, F. X. 1997, MNRAS, 291, 296

Mathis, J. S. 1990, ARA\&A 28, 37

Men'shchikov, A. B., Schertl, D., Tuthill, P. G., Weigelt, G., Yungelson, L. R. 2002, A\&A, 393, 867

Morris, M. R., Sahai, R. \& Claussen, M. 2003, RMxAC 15, 20

Morita, K. 1990, PASJ, 44, 373

Nakashima, J., \& Deguchi, S. 2003, PASJ, 56, 203

Nakashima, J., \& Deguchi, S. 2003, PASJ, 56, 229

Nakashima, J., Deguchi, S., \& Kuno, N. 2004, PASJ, 56, 193

Netzer, N. \& Knapp, G. R. 1987, ApJ, 323, 734

Nordhaus, J. \& Blackman, E. G. 2006, MNRAS, 370,2004

Nyman, L.-Å. Johansson, L. E. B., \& Booth, R. S. 1986, A\&A, 160, 352

Nyman, L.-Å., Hall, P. J., \& Olofsson, H. 1998, A\&AS, 127, 185

Sahai, R. Te Lintel-Hekkert, P., Morris, M., Zijlstra, A., \& Likkel, L. 1999, ApJ, 514, L15S

Sevenster, M. N., van Langevelde, H. J., Moody, R. A., Chapman, J. M., Habing, H. J., \& Killeen, N. E. B. 2001, A\&A, 366, 481

Sevenster, M. N. 2002, AJ, 123, 2788

Schlegel, D. J., Finkbeiner, D. P., Davis, M. 1998, ApJ, 500, 525

Skrutskie, M. F., Stiening, R., Cutri, R., Beichman, C., Capps, R., Carpenter, J., Chester, J., Elias, J. et al. 2000 (The 2MASS Team) http://www.ipac.caltech.edu/2mass/overview/ 2massteam.html
Suh, K-W, \& Jones, T. J. 1997, ApJ, 479, 918

Suh, K.-W. \& Kim, H.-Y. 2002, A\&A, 391, 665

te Lintel Hekkert, P., Caswell, J. L., Habing, H. J., Haynes, R. F., \& Norris, R. P. 1991, A\&AS, 90,327

Tylenda, R. \& Soker, N. 2006, A\&A, 451, 223

van der Veen, W. E. C. J. \& Habing, H. J. 1988, A\&A, 194, 125

van Hoof, P. A. M., Oudmaijer, R. D., \& Waters, L. B. F. M 1997, MNRAS, 289, 371

Verstraete, L., Pech, C., Moutou, C., Sellgren, K., Wright, C. M., Giard, M., Léger, A., Timmermann, R., \& Drapatz, S. 2001, A\&A, 372, 981

Weintraub, D. A. 1990, ApJS, 74, 575

Wilson, W. J. \& Barrett, A. H. 1968, Science, 161, 778

This 2-column preprint was prepared with the AAS LATEX macros v5.2. 
TABLE 1

OBJECTS INVESTIGATED.

\begin{tabular}{|c|c|c|c|c|c|c|c|c|}
\hline Source & MSX6C name & $\begin{array}{l}\text { R.A. } \\
(\mathrm{h} \mathrm{m} \mathrm{s})\end{array}$ & $\begin{array}{l}\text { Dec } \\
\left(0^{\prime \prime \prime \prime}\right)\end{array}$ & $F_{\mathrm{C}}$ & $\mathrm{Cac}$ & Cce & $\begin{array}{c}\mathrm{SiO} \mathrm{H}_{2} \mathrm{O} \mathrm{OH} \\
\left(\text { detection }^{a}\right)\end{array}$ & $\mathrm{OH}$ name \\
\hline $18034-1858$ & G011.0064+00.9220 & 180625.3 & -185744 & 8.8 & 0.194 & 0.186 & $\mathrm{n} \mathrm{n}-$ & \\
\hline $18100-1915$ & G011.5218-00.5826 & $\begin{array}{lll}18 & 13 & 03.1\end{array}$ & $-1914 \quad 19$ & 13.1 & 0.167 & 0.173 & $\mathrm{y} n \mathrm{y}^{3}$ & OH11.52-0.58 \\
\hline $18135-1456$ & G015.7005+00.7707 & $\begin{array}{lll}18 & 16 & 25.7\end{array}$ & -145515 & 26.1 & 0.752 & 0.498 & $\mathrm{y}^{1} \mathrm{y}^{2} \mathrm{y}^{3}$ & $\mathrm{OH} 15.7+0.8$ \\
\hline $18161-1713$ & G013.9883-00.8613 & 181901.7 & $-17 \quad 1207$ & 25.3 & 0.283 & 0.083 & $\mathrm{n} \quad \mathrm{n} \mathrm{n}^{10}$ & \\
\hline $18182-1504$ & G016.1169-00.2903 & 182107.0 & -150322 & 48.5 & 0.190 & 0.268 & $y \quad y^{4} y^{3}$ & OH16.1-0.3 \\
\hline $18198-1249$ & G018.2955+00.4291 & 182243.1 & -124742 & 10.1 & 0.242 & 0.340 & $y n^{4} y^{3}$ & $\mathrm{OH} 18.30+0.43$ \\
\hline $18212-1227$ & G018.7683+00.3016 & 182405.3 & $-12 \quad 2612$ & 3.6 & 0.863 & 0.601 & $n \quad n \quad y^{3}$ & $\mathrm{OH} 18.8+0.3$ \\
\hline $18241-1010$ & G021.1164+00.7775 & 182650.6 & -100819 & 12.4 & 0.176 & 0.099 & $y-n^{10}$ & \\
\hline $18245-1248$ & G018.8384-00.5622 & 182721.2 & -124642 & 1.1 & 0.252 & 0.076 & $y^{5}--$ & \\
\hline $18257-1000$ & G021.4566+00.4911 & 182831.0 & -095815 & 9.6 & 0.172 & 0.305 & $y y^{14} y^{3}$ & ОН021.457+00.491 \\
\hline $18286-0959$ & G021.7964-00.1273 & 183122.9 & -095720 & 45.0 & 0.181 & -0.129 & $\mathrm{n} \quad \mathrm{y} \quad \mathrm{y}^{3}$ & OH021.797-00.127 \\
\hline $18298-0904$ & G022.7482+00.0248 & 183237.0 & -090230 & 18.3 & 0.173 & -0.176 & $\mathrm{n} n-$ & \\
\hline $18325-0721$ & G024.5814+00.2245 & 183519.1 & -071923 & 4.7 & 0.178 & 0.145 & $\mathrm{n} \quad \mathrm{n} \quad \mathrm{y}^{3}$ & $\mathrm{OH} 24.6+0.2$ \\
\hline $18327-0645$ & G025.1301+00.4841 & 183524.3 & -064259 & 10.1 & 0.349 & -0.099 & $\mathrm{n} \mathrm{n}-$ & \\
\hline $18407-0358$ & G028.5203-00.0141 & 184325.8 & -035555 & 3.8 & 0.225 & 0.197 & $\mathrm{y} n \mathrm{y}^{3}$ & $\mathrm{OH} 28.5-0.0$ \\
\hline $18424-0509$ & G027.6621-00.9179 & 184504.9 & -050628 & 14.5 & 0.111 & -0.146 & $\mathrm{n} n-$ & \\
\hline $18444-0359$ & G028.9304-00.8287 & 184705.2 & -035621 & 7.8 & 0.166 & -0.135 & $\mathrm{n} \quad \mathrm{n}-$ & \\
\hline $18450-0148$ & G030.9441+00.0350 & 184741.2 & -014511 & 23.9 & 0.978 & 0.519 & $y^{1} y^{8} y^{3}$ & $\mathrm{OH} 31.8+0.0, \mathrm{~W} 43 \mathrm{~A}$ \\
\hline $18460-0151$ & G031.0126-00.2195 & 184843.0 & -014830 & 14.7 & 0.205 & 0.176 & $\mathrm{n} \quad \mathrm{y} \quad \mathrm{y}^{3}$ & ОН $30.1-0.2$ \\
\hline $18460-0254$ & G030.0908-00.6866 & $1848 \quad 42.0$ & -025029 & 127.9 & 0.184 & 0.261 & $y^{7} y^{14} y^{3}$ & OH30.1-0.7 \\
\hline $18488-0107$ & G031.9844-00.4849 & 185126.2 & -010352 & 31.1 & 0.177 & 0.196 & $\mathrm{y}^{1} \mathrm{y}^{14} \mathrm{y}^{3}$ & OH $32.0-0.5$ \\
\hline $18509-0018$ & G032.9524-00.5687 & 185330.0 & -001428 & 24.9 & 0.211 & 0.122 & $\mathrm{y}^{1}-\mathrm{y}^{3}$ & ОН $32.95-0.57$ \\
\hline $18517+0037$ & G033.8728-00.3350 & 185420.8 & +004105 & 25.9 & 0.244 & 0.187 & $y^{1} y^{9} y^{3}$ & OH033.873-00.335 \\
\hline $18596+0315$ & G037.1185-00.8473 & 190206.3 & +032016 & 2.9 & 0.784 & 0.501 & $\mathrm{n} n \mathrm{y}^{3}$ & OH $37.1-0.8$ \\
\hline $19006+0624$ & G040.0220+00.3818 & 190303.4 & +062854 & 2.2 & 0.276 & 0.060 & $y^{1} y^{14} y^{10}$ & $\mathrm{OH} 40.02+0.38$ \\
\hline $19011+0638$ & G040.2793+00.3766 & 190333.1 & +064229 & 11.4 & 0.297 & 0.206 & $\mathrm{n} \mathrm{n}-$ & \\
\hline $19025+0702$ & G040.8005+00.2455 & 190459.0 & +070640 & 6.9 & 0.354 & 0.068 & $\mathrm{n}--$ & \\
\hline $19087+1006$ & G044.2404+00.3090 & 191110.0 & +101137 & 3.0 & 0.594 & 0.259 & $\mathrm{n} \quad \mathrm{n} \quad \mathrm{n}^{12}$ & \\
\hline $19112+1220$ & G046.4992+00.8092 & 191337.4 & +122539 & 5.2 & 0.383 & 0.058 & $\mathrm{n} \quad \mathrm{n} \quad \mathrm{n}^{10}$ & \\
\hline $19114+0920$ & G043.8675-00.6247 & 191349.4 & +092551 & 10.5 & 0.157 & -0.172 & $\mathrm{n} \quad \mathrm{n} \quad \mathrm{n}^{10}$ & \\
\hline $19180+1230$ & G047.4257-00.5624 & 192022.0 & +123624 & 6.1 & 0.231 & -0.123 & $\mathrm{n}--$ & \\
\hline $19254+1631$ & G051.8042-00.2247 & 192742.1 & +163725 & 22.1 & 0.226 & 0.276 & $y^{1}-y^{13}$ & OH51.8-0.2 \\
\hline $19254+1724$ & G052.5814+00.2014 & 192741.1 & +173036 & 3.4 & 0.675 & 0.273 & $\mathrm{n}-\mathrm{n}^{10}$ & \\
\hline $19266+1815$ & G053.4614+00.3547 & 192853.1 & +182123 & 8.8 & 0.168 & -0.168 & $\mathrm{n}--$ & \\
\hline $19275+1702$ & G052.5042-00.4085 & 192946.5 & +170901 & 9.3 & 0.185 & -0.136 & $\mathrm{n} \quad \mathrm{n}-$ & \\
\hline $19440+2251$ & G059.4784-00.8969 & $1946 \quad 09.2$ & +225924 & 17.4 & 0.153 & 0.258 & $y^{1} n^{8} y^{11}$ & OH59.48-0.90 \\
\hline G014.2758 & G014.2758-00.1602 & 181701.0 & -163700 & 6.1 & 0.183 & 0.122 & $\mathrm{n} \mathrm{n}-$ & \\
\hline G017.3913 & G017.3913-00.2891 & 182335.0 & -135549 & 5.7 & 0.080 & 0.240 & $y n^{6} y^{3}$ & OH17.4-0.3 \\
\hline G022.0425 & G022.0425-00.6084 & 183334.6 & -095736 & 1.2 & 0.628 & 0.632 & $n \quad y^{2} y^{3}$ & OH $22.1-0.6$ \\
\hline G024.6610 & G024.6610+00.0868 & 183557.5 & -071858 & 3.1 & 0.218 & 0.324 & $y n^{6} y^{3}$ & $\mathrm{OH} 24.7+0.1$ \\
\hline G027.8433 & G027.8433-00.1060 & 184230.9 & -043435 & 7.1 & 0.081 & -0.207 & $\mathrm{n} \quad \mathrm{n}-$ & \\
\hline
\end{tabular}

a"y" or "n" indicates the detection or nondetection in this paper, othewise noted. References: ${ }^{1}$ : Nakashima \& Deguchi (2003b), 2: Engels et al. (1986), ${ }^{3}$ : Sevenster et al. (2001), ${ }^{4}$ : Gomez et al. (1990), ${ }^{5}$ : Izumiura et al. (1999), ${ }^{6}$ : Nyman et al. (1986), ${ }^{7}$. : Enman et al. (1998), ${ }^{8}:$ : Likkel et al. $(1992),{ }^{9}:$ Engels \& Lewis $(1996),{ }^{10}$ : te Lintel-Hekkert et al. ${ }^{(1991)},{ }^{11}:$ Eder et al. $(1988),{ }^{12}$ : Lewis et al. (1987), ${ }^{13}$ : Chengalur et al. (1993), ${ }^{14}$ : Deacon et al. (2001). 
TABLE 2

Objects identified with Spitzer-Glimpse Survey.

\begin{tabular}{|c|c|c|c|c|c|c|c|c|c|c|}
\hline $\begin{array}{l}\text { Source } \\
\text { (IRAS or MSX) }\end{array}$ & $\begin{array}{l}l \\
(\circ)\end{array}$ & $\begin{array}{c}b \\
(\circ)\end{array}$ & $\begin{array}{r}F_{3.6} \\
(\mathrm{mJy})\end{array}$ & $\begin{array}{r}F_{4.5} \\
(\mathrm{mJy})\end{array}$ & $\begin{array}{r}F_{5.8} \\
(\mathrm{mJy})\end{array}$ & $\begin{array}{r}F_{8.0} \\
(\mathrm{mJy})\end{array}$ & $\begin{array}{r}{[3.6]} \\
-[5.8] \\
\end{array}$ & $\begin{array}{r}{[3.6]} \\
-[12] \\
\end{array}$ & $A_{K}$ & $f_{c}$ \\
\hline $18034-1858$ & +11.0061 & +0.9222 & 29.9 & 308.8 & 1617.0 & - & 5.30 & 8.74 & 1.50 & 7.6 \\
\hline $18135-1456$ & +15.7011 & +0.7706 & 26.2 & 166.6 & 1033.0 & - & 4.95 & 10.06 & 1.74 & 15.9 \\
\hline $18161-1713$ & +13.9884 & -0.8611 & 264.8 & 1988.0 & 11510.0 & - & 5.06 & 7.52 & 3.33 & 3.2 \\
\hline $18182-1504$ & +16.1173 & -0.2907 & 512.4 & - & 14120.0 & - & 4.57 & 7.51 & 5.64 & 2.6 \\
\hline $18241-1010$ & +21.1166 & +0.7775 & 676.4 & 2918.0 & 9559.0 & - & 3.84 & 5.73 & 1.65 & 1.3 \\
\hline $18245-1248$ & +18.8387 & -0.5622 & 81.2 & 447.1 & 1468.0 & 1750.0 & 4.11 & 5.40 & 4.91 & 0.8 \\
\hline $18257-1000$ & +21.4565 & +0.4911 & 434.1 & - & - & - & & 5.93 & 2.63 & 1.4 \\
\hline $18286-0959$ & +21.7972 & -0.1272 & 541.6 & - & - & - & & 7.37 & 7.71 & 2.0 \\
\hline $18298-0904$ & +22.7483 & +0.0252 & 231.0 & - & 3409.0 & - & 3.89 & 7.31 & 7.15 & 2.1 \\
\hline $18325-0721$ & +24.5814 & +0.2243 & 198.1 & 600.7 & 2845.0 & - & 3.86 & 6.01 & 11.48 & 0.3 \\
\hline $18460-0151$ & +31.0124 & -0.2194 & 23.9 & 374.8 & 2598.0 & - & 6.06 & 9.54 & 8.23 & 6.7 \\
\hline $18460-0254$ & +30.0910 & -0.6865 & 558.9 & 5860.0 & - & - & & 8.47 & 3.35 & 5.5 \\
\hline $18488-0107$ & +31.9845 & -0.4853 & 778.6 & 6270.0 & 18690.0 & - & 4.42 & 6.57 & 2.70 & 2.0 \\
\hline $18509-0018$ & +32.9528 & -0.5689 & 273.0 & 1198.0 & 3568.0 & 3952.0 & 3.76 & 7.47 & 1.59 & 3.6 \\
\hline $18517+0037$ & +33.8727 & -0.3353 & 107.9 & - & 4579.0 & - & 5.03 & 8.52 & 3.62 & 5.6 \\
\hline $18596+0315$ & +37.1184 & -0.8474 & 9.9 & 49.7 & 217.3 & 806.0 & 4.32 & 8.73 & 1.54 & 7.5 \\
\hline $19006+0624$ & +40.0224 & +0.3813 & 85.7 & 484.3 & 1566.0 & - & 4.12 & 6.09 & 2.76 & 1.5 \\
\hline $19011+0638$ & +40.2794 & +0.3761 & 84.9 & 939.7 & 5077.0 & - & 5.41 & 7.89 & 2.71 & 4.2 \\
\hline $19025+0702$ & +40.8006 & +0.2452 & 22.1 & 187.6 & 1009.0 & 4079.0 & 5.11 & 8.80 & 2.54 & 7.2 \\
\hline $19087+1006$ & +44.2406 & +0.3086 & 00.9 & 14.7 & 146.5 & 971.8 & 6.49 & 11.37 & 3.80 & 28.3 \\
\hline $19112+1220$ & +46.4995 & +0.8093 & 01.6 & 23.5 & 193.8 & 1029.0 & 6.17 & 11.35 & 1.88 & 32.9 \\
\hline $19114+0920$ & +43.8677 & -0.6248 & 192.3 & 865.6 & 2582.0 & 1809.0 & 3.78 & 6.91 & 2.76 & 2.4 \\
\hline G024.6610 & +24.6608 & +0.0867 & 6.1 & 130.0 & 990.5 & - & 6.49 & 9.33 & 11.05 & 4.7 \\
\hline G027.8433 & +27.8430 & -0.1061 & 236.1 & 811.8 & 2035.0 & 2720.0 & 3.30 & 6.26 & 6.30 & 1.2 \\
\hline
\end{tabular}


Table 3: Detections by the $\mathrm{SiO}$ or $\mathrm{H}_{2} \mathrm{O}$ maser line.

\begin{tabular}{|c|c|c|c|c|c|}
\hline Source & Transition & $\begin{array}{r}V_{l s r} \\
\left(\mathrm{~km} \mathrm{~s}^{-1}\right) \\
\end{array}$ & $\begin{array}{l}T a^{*} \\
(\mathrm{~K})\end{array}$ & $\begin{array}{r}\text { line flux } \\
\left(\mathrm{K} \mathrm{km} \mathrm{s}^{-1}\right) \\
\end{array}$ & $\begin{array}{r}\mathrm{rms} \\
(\mathrm{K})\end{array}$ \\
\hline \multirow[t]{3}{*}{$18100-1915$} & $\mathrm{SiO} J=1-0 v=1$ & & & & 0.121 \\
\hline & $\mathrm{SiO} J=1-0 v=2$ & 16.5 & 1.202 & 2.087 & 0.114 \\
\hline & $\mathrm{H}_{2} \mathrm{O} \quad 6_{16}-5_{23}$ & & & & 0.109 \\
\hline \multirow[t]{5}{*}{$18182-1504$} & $\mathrm{SiO} J=1-0 v=0$ & 18.3 & 0.650 & 2.536 & 0.153 \\
\hline & $\mathrm{SiO} J=1-0 v=1$ & 22.9 & 16.661 & 45.758 & 0.166 \\
\hline & $\mathrm{SiO} J=1-0 v=2$ & 22.5 & 41.500 & 130.23 & 0.168 \\
\hline & $\mathrm{SiO} J=1-0 v=3$ & 22.5 & 10.434 & 28.839 & 0.173 \\
\hline & ${ }^{29} \mathrm{SiO} J=1-0 v=1$ & 23.6 & 1.093 & 2.623 & 0.151 \\
\hline \multirow[t]{2}{*}{$18198-1249$} & $\mathrm{SiO} J=1-0 v=1$ & & & & 0.104 \\
\hline & $\mathrm{SiO} J=1-0 v=2$ & 48.2 & 0.579 & 0.429 & 0.107 \\
\hline \multirow{2}{*}{$18241-1010$} & $\mathrm{SiO} J=1-0 v=1$ & & & & 0.084 \\
\hline & $\mathrm{SiO} J=1-0 v=2$ & 116.3 & 0.620 & 2.795 & 0.085 \\
\hline \multirow{2}{*}{$18257-1000$} & $\mathrm{SiO} J=1-0 v=1$ & & & & 0.097 \\
\hline & $\mathrm{SiO} J=1-0 v=2$ & 114.9 & 0.781 & 1.427 & 0.097 \\
\hline \multirow[t]{5}{*}{$18286-0959$} & $\mathrm{SiO} J=1-0 v=1$ & - & - & & 0.123 \\
\hline & $\mathrm{SiO} J=1-0 v=2$ & & & & 0.118 \\
\hline & $\mathrm{H}_{2} \mathrm{O} 66_{16}-5_{23}$ & -14.7 & 3.863 & 166.3 & 0.043 \\
\hline & $\mathrm{SiO} J=2-1 v=1$ & & & & 0.065 \\
\hline & $\mathrm{HCO}^{+} J=1-0$ & & & & 0.068 \\
\hline \multirow{3}{*}{$18407-0358$} & $\mathrm{SiO} J=1-0 v=1$ & 106.5 & 0.572 & 1.457 & 0.108 \\
\hline & $\mathrm{SiO} J=1-0 v=2$ & 107.3 & 0.921 & 2.126 & 0.097 \\
\hline & $\mathrm{H}_{2} \mathrm{O} \quad 6_{16}-5_{23}$ & & & - & 0.087 \\
\hline \multirow[t]{5}{*}{$18460-0151$} & $\mathrm{SiO} J=1-0 v=1$ & 一 & - & - & 0.100 \\
\hline & $\mathrm{SiO} J=1-0 v=2$ & - & - & 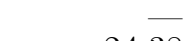 & 0.084 \\
\hline & $\mathrm{H}_{2} \mathrm{O} 6_{16}-5_{23}$ & 117.0 & 4.881 & 24.38 & 0.050 \\
\hline & $\mathrm{SiO} J=2-1 v=1$ & & & & 0.069 \\
\hline & $\mathrm{HCO}^{+} J=1-0$ & - & - & - & 0.084 \\
\hline \multirow[t]{2}{*}{ G017.3913 } & $\mathrm{SiO} J=1-0 v=1$ & - & 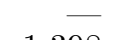 & 5 & 0.119 \\
\hline & $\mathrm{SiO} J=1-0 v=2$ & 28.7 & 1.398 & 4.106 & 0.117 \\
\hline \multirow{2}{*}{ G024.6610 } & $\mathrm{SiO} J=1-0 v=1$ & & & & 0.103 \\
\hline & $\mathrm{SiO} J=1-0 v=2$ & 57.7 & 1.247 & 3.662 & 0.101 \\
\hline
\end{tabular}

Table 4: Negative results for the $\mathrm{SiO}$ and $\mathrm{H}_{2} \mathrm{O}$ maser line search.

\begin{tabular}{lccc}
\hline \hline Source & $\begin{array}{c}\mathrm{rms} \\
(\mathrm{SiO} v=1) \\
(\mathrm{K})\end{array}$ & $\begin{array}{c}\mathrm{rms} \\
(\mathrm{SiO} v=2) \\
(\mathrm{K})\end{array}$ & $\begin{array}{c}\mathrm{rms}^{\mathrm{a}} \\
\left(\mathrm{H}_{2} \mathrm{O}\right) \\
(\mathrm{K})\end{array}$ \\
\hline $18034-1858$ & 0.115 & 0.114 & 0.096 \\
$18161-1713$ & 0.119 & 0.115 & 0.107 \\
$18212-1227$ & 0.096 & 0.095 & 0.086 \\
$18298-0904$ & 0.097 & 0.105 & 0.075 \\
$18325-0721$ & 0.075 & 0.073 & 0.087 \\
$18327-0645$ & 0.099 & 0.097 & 0.088 \\
$18424-0509$ & 0.357 & 0.396 & 0.088 \\
$18444-0359$ & 0.126 & 0.121 & 0.113 \\
$18596+0315$ & 0.117 & 0.115 & 0.086 \\
$19011+0638$ & 0.113 & 0.102 & 0.084 \\
$19025+0702$ & 0.111 & 0.113 & - \\
$19087+1006$ & 0.077 & 0.075 & 0.080 \\
$19112+1220$ & 0.088 & 0.084 & 0.082 \\
$19114+0920$ & 0.086 & 0.086 & 0.151 \\
$19180+1230$ & 0.071 & 0.073 & - \\
$19254+1724$ & 0.094 & 0.082 & - \\
$19266+1815$ & 0.077 & 0.071 & - \\
$19275+1702$ & 0.094 & 0.090 & 0.093 \\
$\mathrm{G} 014.2758$ & 0.109 & 0.109 & 0.099 \\
$\mathrm{G} 022.0425$ & 0.075 & 0.078 & - \\
$\mathrm{G} 027.8433$ & 0.103 & 0.099 & 0.062 \\
\hline
\end{tabular}

${ }^{a}$ Dash indicates not observed. 
Table 5: Observation summary for IRAS 19027+0517.

\begin{tabular}{lccccc}
\hline \hline Transition & $\begin{array}{c}\text { rest freq. } \\
(\mathrm{GHz})\end{array}$ & $\begin{array}{c}V_{l s r} \\
\left(\mathrm{~km} \mathrm{~s}^{-1}\right)\end{array}$ & $\begin{array}{c}T_{a}^{*} \\
(\mathrm{~K})\end{array}$ & $\begin{array}{c}\text { line flux } \\
\left(\mathrm{km} \mathrm{s}^{-1} \mathrm{~K}\right)\end{array}$ & $\begin{array}{c}\text { rms } \\
(\mathrm{K})\end{array}$ \\
\hline $\mathrm{H}_{2} \mathrm{O} 6_{16}-5_{23}$ & 22.23508 & - & - & - & 0.073 \\
$\mathrm{NH}_{3} 1_{1}-1_{1}$ & 23.694 & - & - & - & 0.063 \\
$\mathrm{NH}_{3} 2_{2}-2_{2}$ & 23.722 & - & - & - & 0.044 \\
$\mathrm{NH}_{3} 3_{3}-3_{3}$ & 23.870 & - & - & - & 0.052 \\
$\mathrm{SiO} J=1-0 v=0$ & 43.42386 & 34.2 & 0.173 & 0.417 & 0.053 \\
$\mathrm{SiO} J=1-0 v=1$ & 43.12208 & 31.7 & 1.288 & 3.348 & 0.058 \\
$\mathrm{SiO} J=1-0 v=2$ & 42.82059 & 31.7 & 1.202 & 3.826 & 0.067 \\
$\mathrm{SiO} J=1-0 v=3$ & 42.51938 & 31.6 & 0.298 & 0.557 & 0.053 \\
$29 \mathrm{SiO} J=1-0 v=1$ & 42.87992 & - & - & - & 0.054 \\
$\mathrm{H} 53 \alpha$ & 42.95197 & - & - & - & 0.062 \\
$\mathrm{SiO} J=2-1 v=1$ & 86.24342 & 32.3 & 0.204 & 0.584 & 0.034 \\
$\mathrm{SiO} J=2-1 v=0$ & 86.84700 & - & - & - & 0.037 \\
$\mathrm{HCN} J=1-0$ & 88.63185 & - & - & - & 0.053 \\
$\mathrm{HCO} J=1-0$ & 89.18852 & - & - & - & 0.061 \\
$\mathrm{CO} J=1-0$ & 115.27120 & $-\mathrm{a}$ & - & - & 0.200 \\
\hline
\end{tabular}

${ }^{a}$ Contaminations at $V_{l s r}=12,46$, and $63 \mathrm{~km} \mathrm{~s}^{-1}$. 


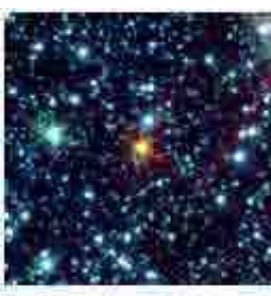

$18034-1858$

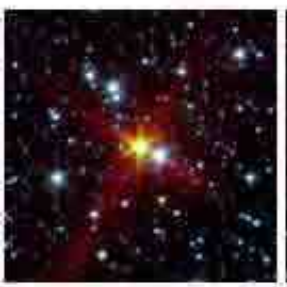

$18182-1504$

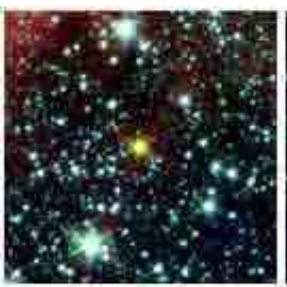

$18245-1248$

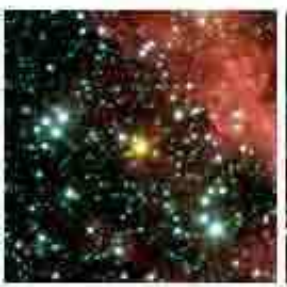

$18325-0721$

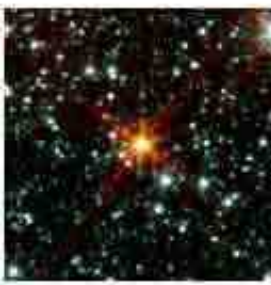

$18444-0359$

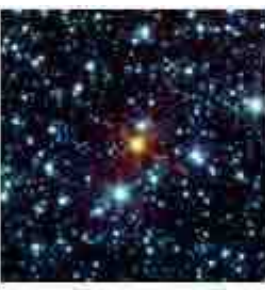

$18100-1915$

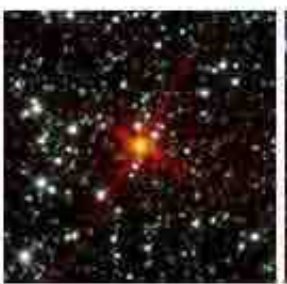

$18198-1249$

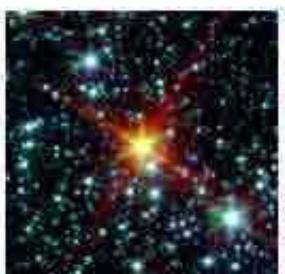

$18257-1000$

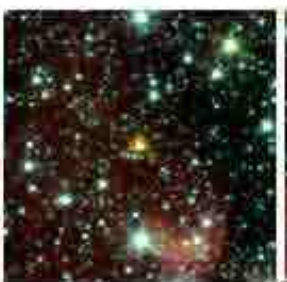

18327-0645

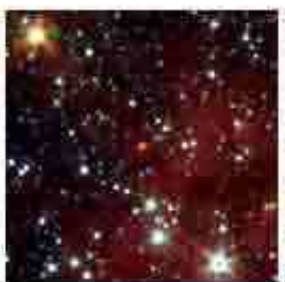

$18450-0148$

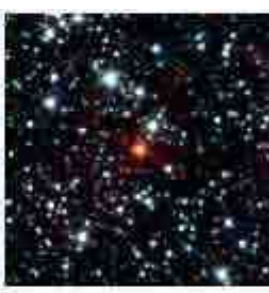

18135-1456

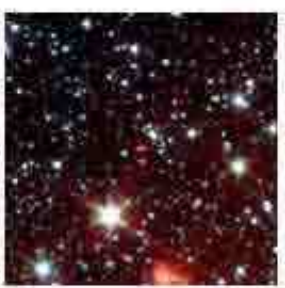

18212-1227

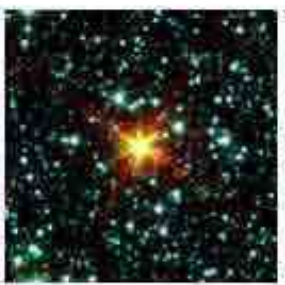

18286-0959

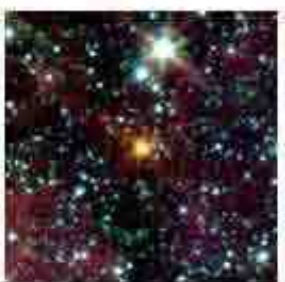

18407-0358

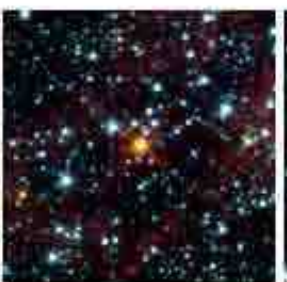

18460-0151

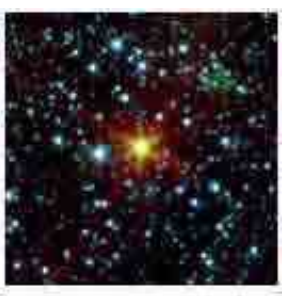

$18161-1713$

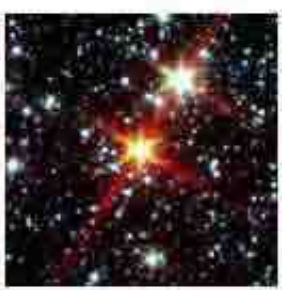

$18241-1010$

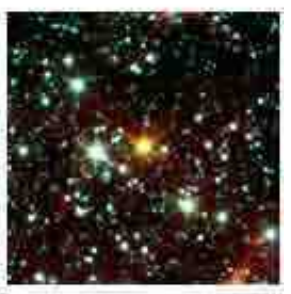

$18298-0904$

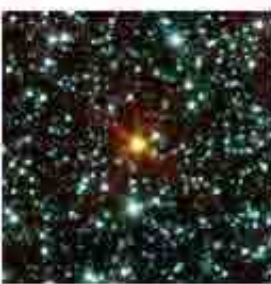

18424-0509

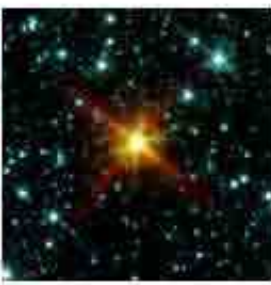

$18460-0254$

Fig. 1.- a. Composite-color images $\left(285^{\prime \prime} \times 285^{\prime \prime}\right)$ of the GLIMPSE field of the sources in Table 1. IRAC 3.6, 4.5 and $5.8 \mu \mathrm{m}$ bands are represented by blue, green, and red colors respectively. Objects are at the center of the images, and the directions of increasing Galactic longitude and latitude are left and up, respectively. 


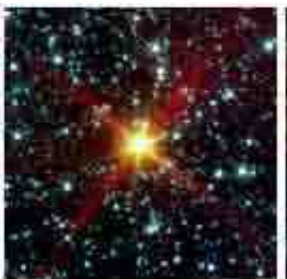

18488-0107

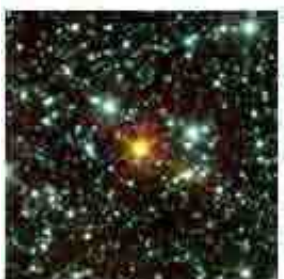

$19006+0624$

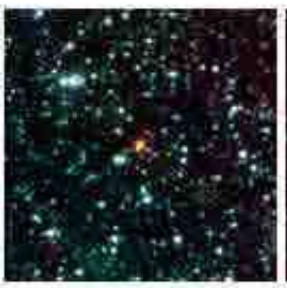

$19112+1220$

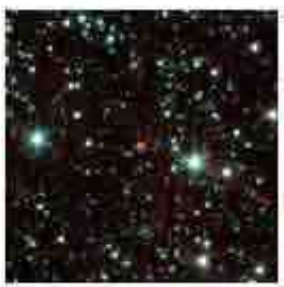

$19254+1724$

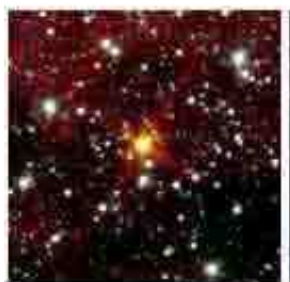

G014:2758-00.1602

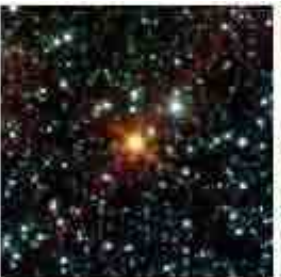

$18509-0018$

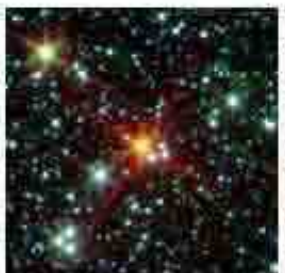

$19011+0638$

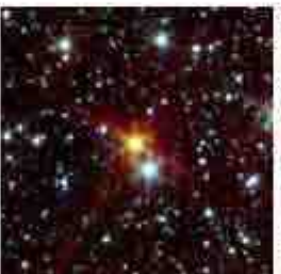

$19114+0920$

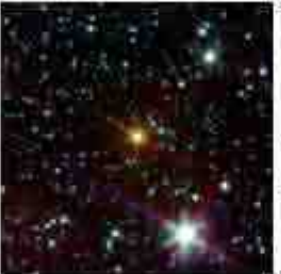

$19266+1815$

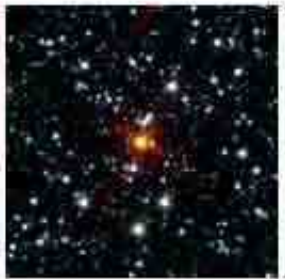

G017.3913-00.2891

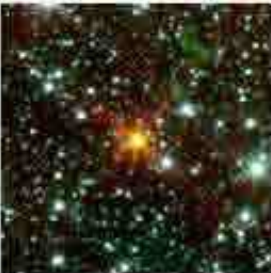

$18517+0037$

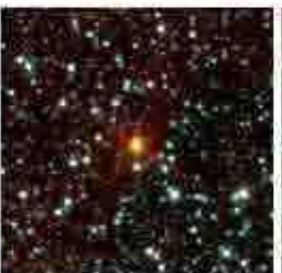

$19025+0702$

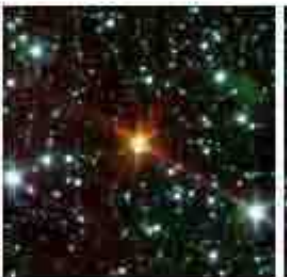

$19180+1230$

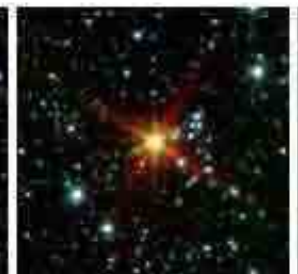

$19275+1702$

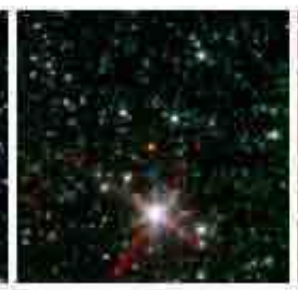

G022.0425-00.6084

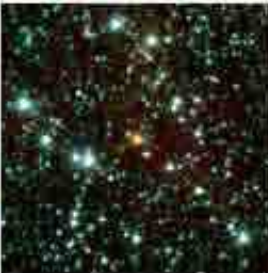

$18596+0315$

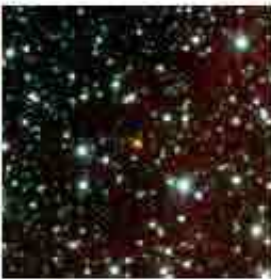

$19087+1006$

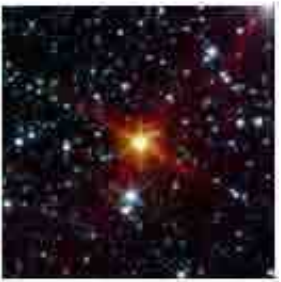

$19254+1631$

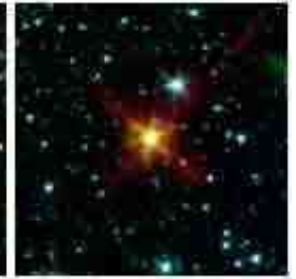

$19440+2251$

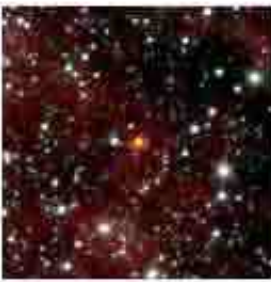

$\mathrm{G} 024.6610+00.0868$

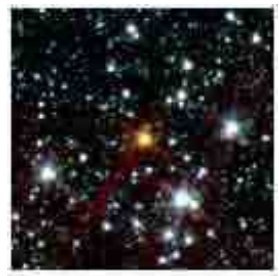

G027.8433-00.1060

Fig. 1.- b. and c. - Continued. 

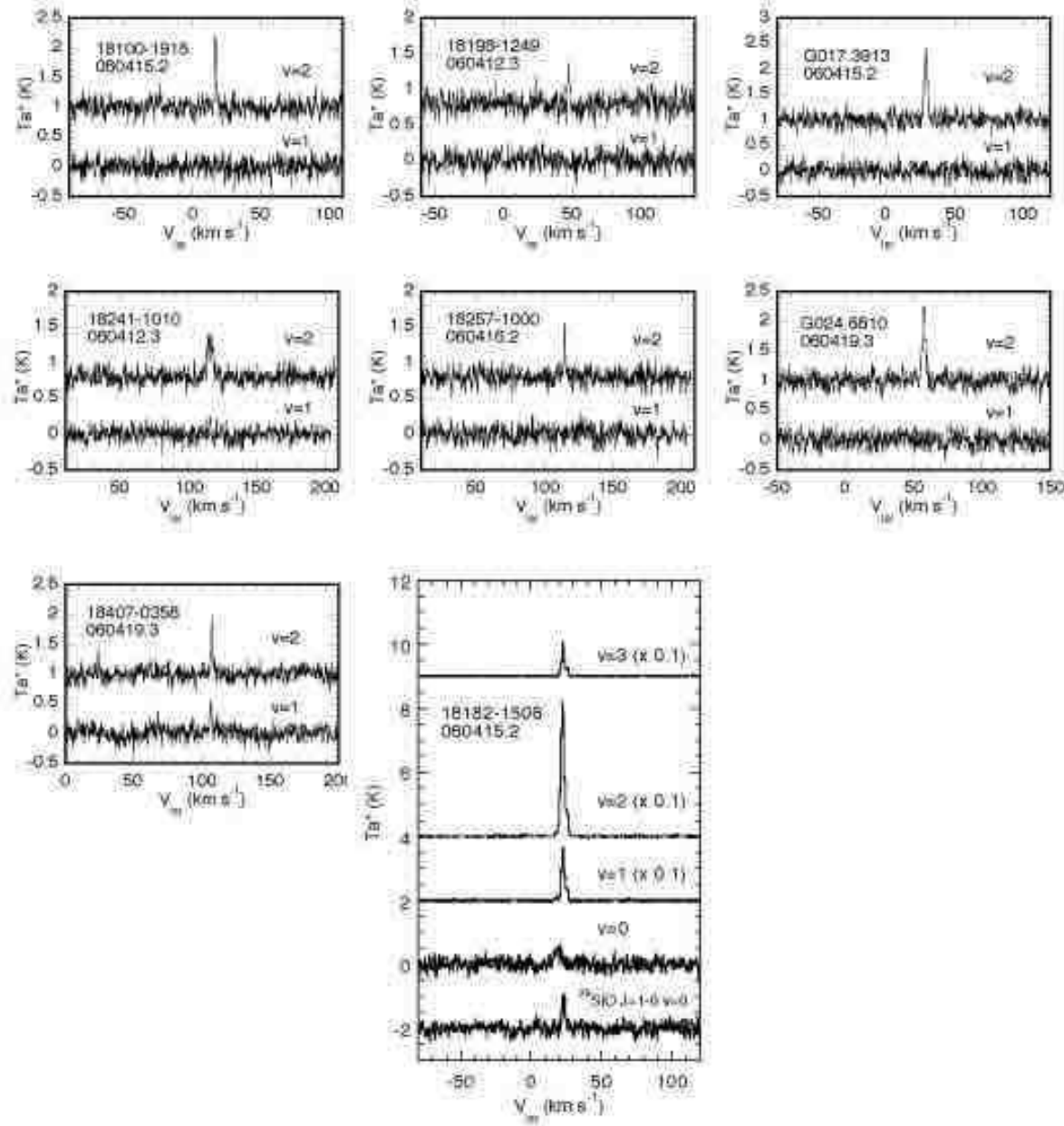

Fig. 2. $-\mathrm{SiO} J=1-0 v=1$ and 2 spectra of the detected sources. For IRAS $18182-1504$ the $\mathrm{SiO} J=1-0$ $v=01,2$, and 3 , and ${ }^{29} \mathrm{SiO} J=1-0 v=0$ spectra are shown. Source name and observed date (in yymmdd.d format) are indicated on the left of each panel. were indicated
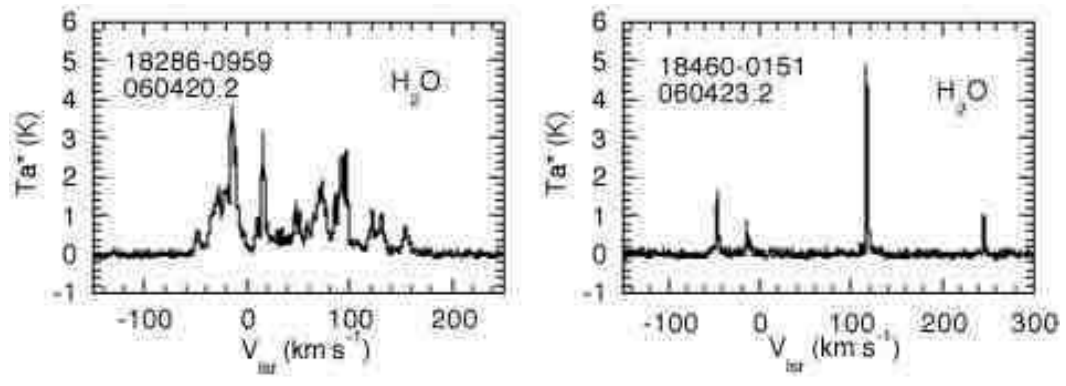

Fig. 3.- $\mathrm{H}_{2} \mathrm{O} 6_{16}-5_{23}$ spectra for the detected sources. Source name and observed date (in yymmdd.d format) are indicated on the left of each panel. 


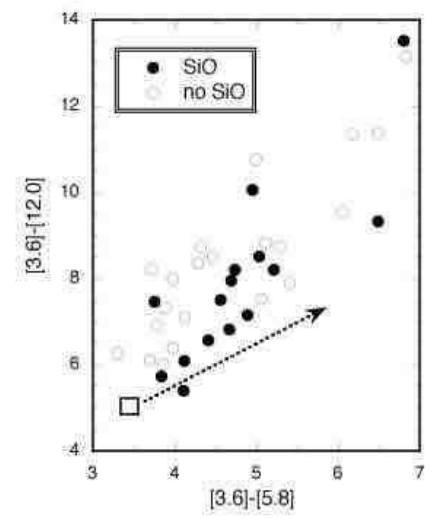

Fig. 4.- Two-color diagram, [3.6] - [12] versus [3.6] - [5.8]. The filled and unfilled circles indicate SiO detection and nondetection, and the square indicates the position of $\mathrm{OH} 127.8+0.0$. The dotted arrow indicates direction of interstellar reddening; the length corresponds to $A_{K}=15$.

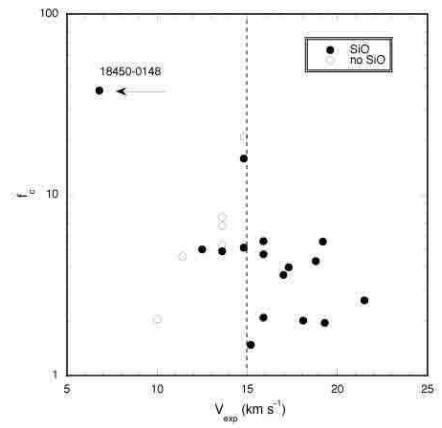

Fig. 5.- Excess factor versus expansion velocity of the $\mathrm{OH} 1612 \mathrm{MHz}$ double-peak objects. Filled and unfilled circles indicate $\mathrm{SiO}$ detection and nondetection. The broken line indicates a separation for the low-mass stars.

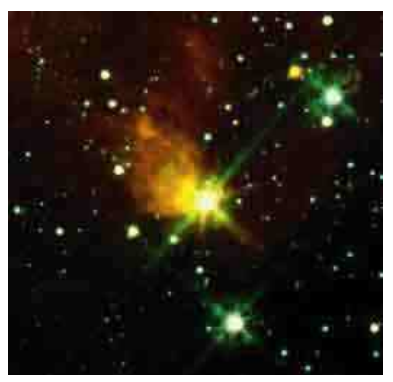

Fig. 6.- Glimpse color-composite image $\left(285^{\prime \prime} \times 285^{\prime \prime}\right)$ of IRAS $19027+0517$ made from 4.5 (blue), 5.8 (green), and 8.0 (red) $\mu \mathrm{m}$ IRAS image. The directions of increasing Galactic longitude and latitude are left and up, respectively. 

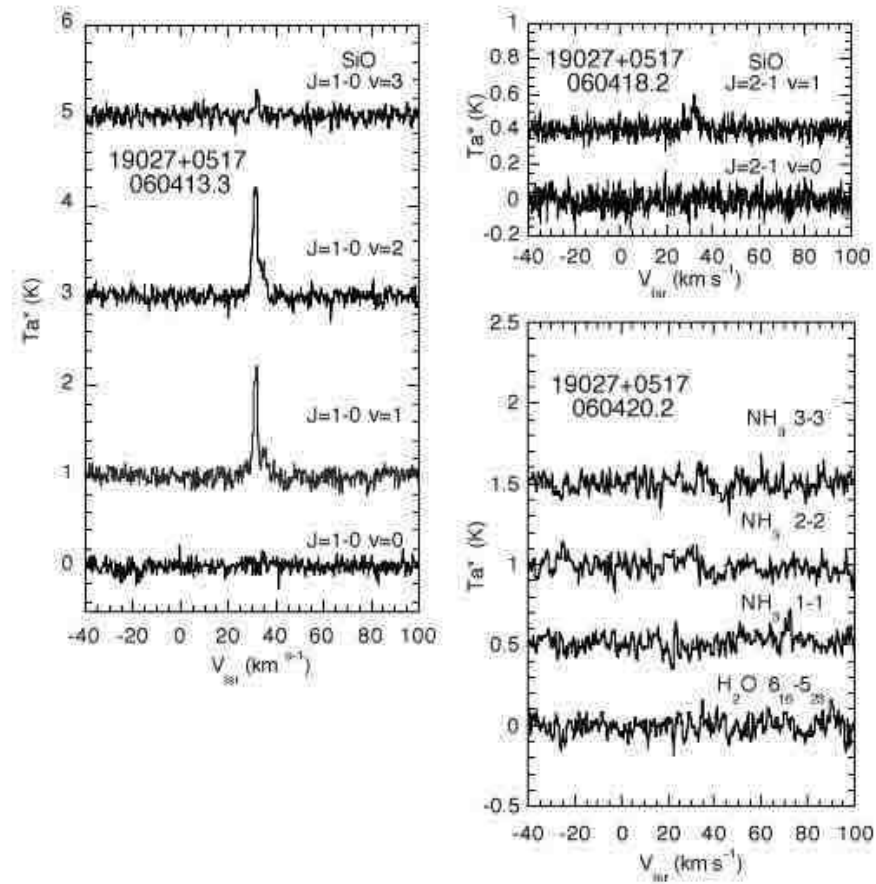

Fig. 7. - $\mathrm{SiO}, \mathrm{H}_{2} \mathrm{O}$ and $\mathrm{NH}_{3}$ spectra (a) $\mathrm{CO} J=1-0$ spectra (b) toward IRAS $19027+0517$. The number between the parenthesis on the left indicates R.A. and Dec. offsets in arcsec from the star position. The top is the spectrum of the bottom (center) subtracted by the average of surrounding 4 positions (middle).

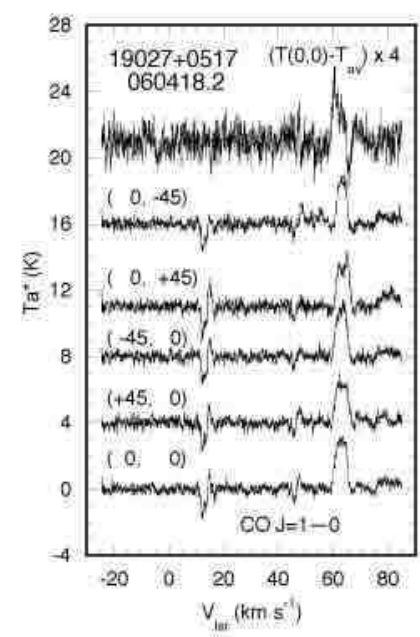

Fig. 8.- CO $J=1-0$ spectra toward IRAS $19027+0517$. The number between the parenthesis on the left indicates R.A. and Dec. offsets in arcsec from the star position. The top is the spectrum of the bottom (center) subtracted by the average of surrounding 4 positions (middle). 